\title{
The efficacy and safety of $0.5 \%$ Levofloxacin versus fortified Cefazolin and Amikacin ophthalmic solution for the treatment of suspected and culture-proven cases of infectious bacterial keratitis: a comparative study
}

\author{
Ngamjit Kasetsuwan ${ }^{\mathrm{a}}$, Pinnita Tanthuvanit ${ }^{\mathrm{b}}$, Usanee Reinprayoon ${ }^{\mathrm{c}}$ \\ ${ }^{a}$ Department of Ophthalmology, Faculty of Medicine, Chulalongkorn University, Bangkok 10330; \\ ${ }^{b}$ Department of Ophthalmology, Faculty of Medicine, Siriraj Hospital, Mahidol University, Bangkok \\ 10700, 'Department of Ophthalmology, King Chulalongkorn Memorial Hospital, Bangkok 10330, \\ Thailand
}

\begin{abstract}
Background: Bacterial keratitis is a major devastating ocular condition that quickly deteriorates the patient's vision. Vigorous and prompt treatment of bacterial keratitis with broad-spectrum antibiotic eye-drops is preferred. Objective: Evaluate the efficacy and safety of $0.5 \%$ Levofloxacin for the treatment of suspected and cultureproven cases of infectious bacterial keratitis in comparison to fortified Cefazolin and Amikacin ophthalmic solution. Materials and methods: Seventy-one eyes from 69 patients suspected of having infectious bacterial keratitis were enrolled in the study. The patients were randomized into two arms, $0.5 \%$ Levofloxacin eye drops (34 eyes) or fortified Cefazolin and Amikacin (37 eyes). Sixty-eight eyes were included in the efficacy analysis. During treatment, on days 2, 7, 14, and 21, the patient's symptoms and signs were scored from grade 0 -3 (absent to severe).

Results: At the end of the treatment, 61 out of 71 eyes completely healed. The resolution of the keratitis was not significantly different between both groups. There were no significant differences in the mean time-duration for the ulcer to heal or for the symptoms and clinical signs to disappear between the two groups. No serious adverse events or side effects from the disease were found. The patients compliance was $80 \%$ based on the self-reported diaries.

Conclusion: The efficacy and safety of $0.5 \%$ topical Levofloxacin was comparable to fortified Cefazolin and Amikacin for the treatment of mild-to-moderate bacterial keratitis. Topical Levofloxacin is far superior because of its availability and patient compliance when used as monotherapy for the treatment of infectious bacterial keratitis.
\end{abstract}

Keywords: Bacterial keratitis, fluoroquinolone, fortified topical antibiotics, Levofloxacin

Infectious keratitis is a serious condition that affects and threatens the patient sight. People infected with keratitis can have permanent visual impairment or visual loss if left untreated [1]. Vigorous and prompt treatment of bacterial keratitis with broadspectrum antibiotic eye drops is preferred because of its ability to penetrate ocular tissues and deliver the drug to its targeted location. Generally, a combination of fortified broad-spectrum antibiotics is considered

Correspondence to: Ngamjit Kasetsuwan, MD, Department of Ophthalmology, Faculty of Medicine, Chulalongkorn University, 1873 Rama IV Road, Pathumwan, Bangkok 10330, Thailand.E-mail:ngamjitk@gmail.com as the gold standard treatment for moderate-to-severe or unresponsive bacterial keratitis [2].

In Thailand, severe corneal ulcers were detected in $17-30 \%$ of patients attending referral centers [1]. The leading pathogen for infectious keratitis is Pseudomonas aeruginosa (60\%-70\%), and followed by Streptococcus pneumonia [1]. If left untreated, most patients would develop complications such as secondary glaucoma, cataract, or endophthalmitis, and consequently have poorer treatment outcomes. As for the initial treatment of infectious bacterial keratitis, monotherapy of fluoroquinolone ophthalmic solution has been widely used because of its great penetrability property and safety profile [3-5]. 
Levofloxacin is one of the third generation fluoroquinolones, developed from isolating the active enantiomer of ofloxacin. It has ability to functionally disrupt the bacterial replication process by binding to both deoxyribonucleic acid (DNA) gyrase and topoisomerase IV [6]. This modification has made gram-positive bacteria, Strep. pneumoniae and Strep.viridans, more vulnerable to this drug [6, 7].

We believe that levofloxacin monotherapy should be beneficial in resource constraint settings due to its availability in a non-persevered-bottle, fewer side effects when compared to fortified treatment, and cheaper price. This drug's ease of use will increase the patient's adherence to the treatment regimen and significantly reduce costs for the treatment of infectious bacterial keratitis. In this study, we compared the efficacy and safety of $0.5 \%$ topical Levofloxacin to that of fortified Cefazolin $(50 \mathrm{mg} / \mathrm{mL})$, and Amikacin $(50 \mathrm{mg} / \mathrm{mL}$ ) ophthalmic solution for the treatment of mild-to-moderate bacterial keratitis.

\section{Materials and methods}

This is a prospective, double-blind, and randomized controlled clinical trial (RTC). This study was approved by the Ethics Committee of Faculty of Medicine, Chulalongkorn University and the Ethics Committee of Siriraj Hospital, Faculty of Medicine, Mahidol University.

\section{Patients}

Patients who were clinically suspected to have mild and moderate bacterial keratitis at the General Eye and Corneal Clinic at the King Chulalongkorn Memorial Hospital and Siriraj Hospital were enrolled into the study. Severity grading of bacterial keratitis was based on the modified Jones criteria [8]. Patients who were allergic to fluoroquinolone, aminoglycoside, penicillin, cephalosporin or benzalkonium chloride and had ocular surface problems (eg. neurotrophic or exposure keratopathy) were excluded from the study. Also, patients with fungal, viral, acanthamoeba or severe infectious bacterial keratitis were excluded. Our study prohibited pregnant or lactating women from participating in the study for obvious reasons. Informed consent was obtained from each subject before entering the study.

\section{Study protocol}

Patients were assigned into either the arm that received $0.5 \%$ levofloxacin or fortified Cefazolin
(50 mg/mL) and Amikacin (50 mg/mL), using a block of four randomization. Both the patients and study physicians were blinded. Both groups received the same number of unidentified bottles. The fortified Cefazolin/Amikacin received two bottles of medications whereas the Levofloxacin received one bottle of medication and one bottle of BSS (Balanced salt solution)

The patient ocular history, systemic diseases, risk factors, and medication use was recorded. Complete ocular examination, including measurements for uncorrected and best-corrected visual acuity, was done on all patients on day 0. Slit-lamp microscopy was used to assess location, size, depth, and characteristics of the infiltration. Epithelial defect was accessed by using fluorescein staining. The anterior chamber reaction was used to detect the level of hypopyon, which was measured in millimeters.

To confirm the clinical diagnosis before starting treatment, corneal specimens were collected from all patients in Amie media. The specimens were sent to the laboratory and stained with Gram stain. Cultures were performed on blood agar, chocolate agar, Sabouraud dextrose agar, and thioglycolate broth.

All patients underwent treatment for infectious bacterial keratitis. The treatment schedule for both groups was exactly the same. Both groups would apply their assigned medications every 10 minutes during the first 30 minutes of treatment and later decreased it to one hour every three days. On days 4-7, one drop was used every two to four hours depending on the patients' clinical responses. After day 7, topical antibiotics were tapered to every six hours and discontinued when there was evidence that the ulcer had completely healed.

During treatment, the patients were followed on days $2,7,14$, and 21. During each visit, the patient's symptoms and signs were assessed using scores from grade 0-3 (absent to severe). Symptoms accessed were for discomfort, pain, tearing, photophobia, and itching. Changes in clinical signs, such as swollen eyelids, chemosis, and conjunctival hyperemia were evaluated using biomicroscopy examination. Those patients whose symptoms worsened or did not respond to the assigned drugs were considered as failures, and subsequently withdrew from the study.

\section{Statistical analysis}

The primary outcome of this study was to assess the resolution of keratitis (healed or not healed). Cured 
or healed ulcer was defined as no corneal infiltration, no sign of inflammation and complete epithelial healing. Treatment failure was defined as those who were unresponsive to their treatment and showed no clinical improvement or worsening of clinical signs within 72 hours. Once the patients were classified as treatment failures, the treating physicians were unmasked so that they could give the appropriate treatment. The secondary outcome measured the time-duration for the ulcer to heal, or for the symptoms and clinical signs to disappear. This was reported as scores for clinical symptoms and sign.

Comparisons between both arms were done using Chi-square test for nominal variations (resolution of keratitis, laterality, sex, risk factors, location, depth, and severity of the ulcers). The time-durations for the ulcer to heal and for the symptoms and clinical signs to disappear were estimated using Kaplan-Meier survival curves. Student t-test was used to compare the mean age of both groups.

\section{Results}

Seventy one eyes from 69 patients were analyzed.
Thirty seven eyes went to the fortified Cefazolin/ Amikacin group and 34 eyes went to the Levofloxacin group. Sixty eight eyes were analyzed for efficacy. Since five cases were lost to follow-up, they were classified as having ulcers that did not heal whereas three cases with positive fungal cultures were excluded from the analysis. Figure 1 shows the flow diagram of participant trial.

Table 1 shows demographic and clinical characteristics at baseline in fortified Cefazolin/ Amikacin and Levofloxacin groups. No statistical differences were detected between the two groups. The characteristics of the ulcers at baseline in both groups are shown in Table 2.

In fortified Cefazolin/Amikacin group, $67.6 \%$ and $32.4 \%$ had mild and moderate corneal ulcer, respectively. As for the Levofloxacin group, 64.7\% and $35.3 \%$ had mild and moderate severity, respectively. In addition, no statistical differences were detected between both groups for clinical signs $(\mathrm{p}=0.99)$ and symptom scores $(\mathrm{p}=0.85$, Table 3$)$ at week 0 .

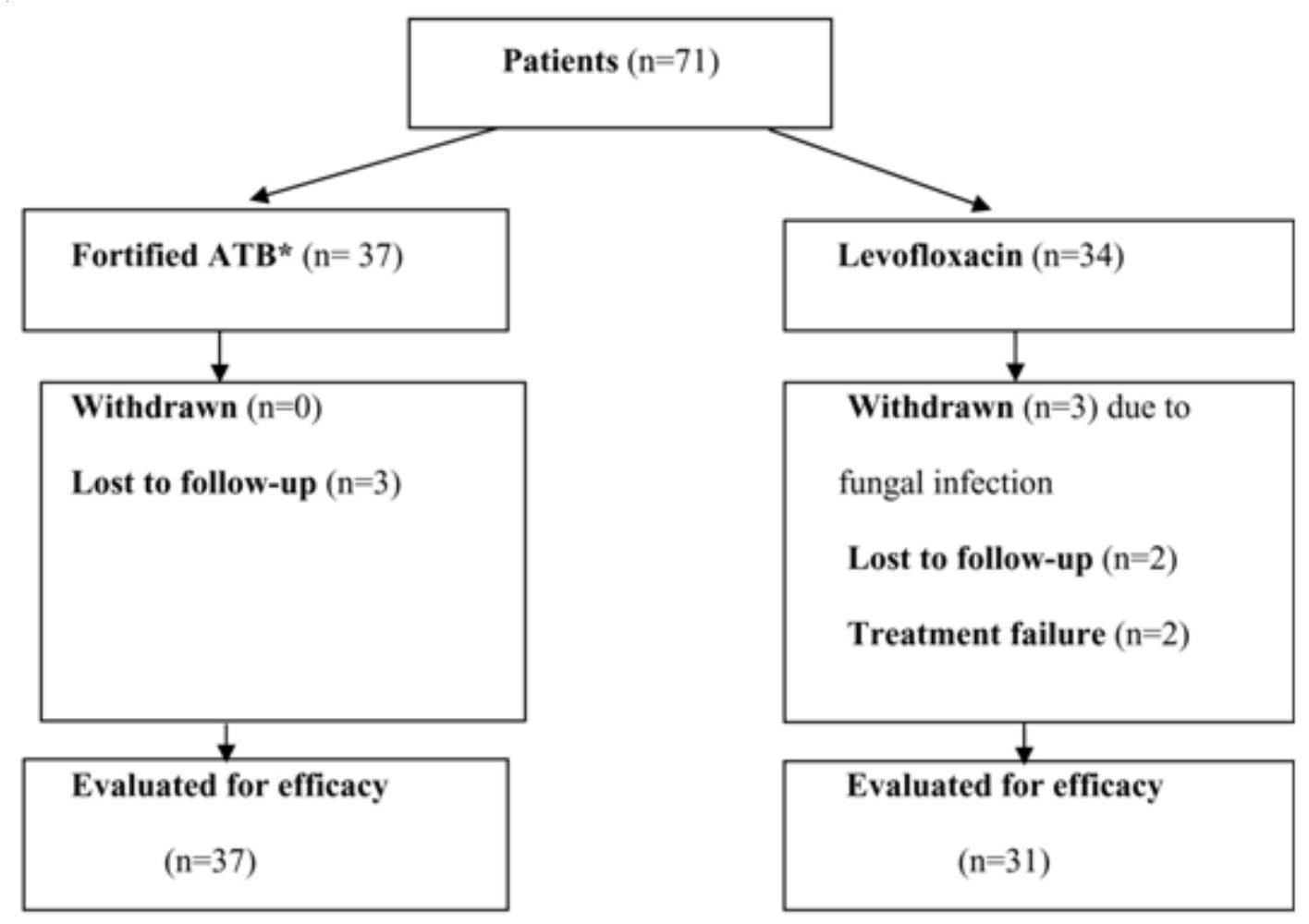

Fig. 1 A randomized controlled trial CONSORT flow diagram (*antibiotics). 
Table 1. Demographic and clinical characteristics of the study population at baseline.

\begin{tabular}{lccc}
\hline & $\begin{array}{c}\text { Fortified } \\
\text { Cefazolin/Amikacin } \\
(\mathbf{n = 3 7 )}\end{array}$ & $\begin{array}{c}\text { Levofloxacin } \\
\text { (n=34) }\end{array}$ & P-value \\
\hline Age & & & \\
$\quad$ Mean \pm SD & $34.4 \pm 15.4$ & 0.97 \\
$\quad$ Range & $7-71$ & $34.6 \pm 18.1$ & \\
Eye $\quad 22(59.5 \%)$ & $9-84$ & 0.42 \\
$\quad$ Right & $15(40.5 \%)$ & $16(47.1 \%)$ & \\
$\quad$ Left & $19(51.4 \%)$ & $18(52.9 \%)$ & 0.86 \\
Gender & $18(48.6 \%)$ & $19(55.9 \%)$ & 0.99 \\
$\quad$ Male & $3.72+2.09$ & $15(44.1 \%)$ & 0.85 \\
$\quad$ Female & $9.54+4.29$ & $5.60+12.03$ & \\
$\quad$ Mean clinical sign scores & & $9.47+3.78$ & \\
$\quad$ Mean symptom scores & 15 & 15 & 0.18 \\
Risk factors & 4 & 5 & \\
$\quad$ Contact lens & 7 & 8 & \\
$\quad$ Ocular allergy & & & \\
$\quad$ Trauma & & & \\
\hline
\end{tabular}

Table 2. Baseline ulcer characteristics of patients enrolled into the trial.

\begin{tabular}{|c|c|c|c|}
\hline & $\begin{array}{c}\text { Fortified } \\
\text { Cefazolin/Amikacin } \\
(\mathbf{n}=37)\end{array}$ & $\begin{array}{l}\text { Levofloxacin } \\
\quad(n=34)\end{array}$ & P-value \\
\hline \multicolumn{4}{|l|}{ Severity of ulcer } \\
\hline Mild & $25(67.6 \%)$ & $22(64.7 \%)$ & 0.99 \\
\hline Moderate & $12(32.4 \%)$ & $12(35.3 \%)$ & \\
\hline \multicolumn{4}{|l|}{ Infiltrative size } \\
\hline Median (minum-maximum) & $1.2(0.9-25)$ & $2.1(0.49-36)$ & 0.81 \\
\hline Size of epithelial defect & $1(0-25)$ & $1.72(0.49-36)$ & 0.93 \\
\hline \multicolumn{4}{|l|}{ Location of ulcer } \\
\hline Axial & 17 & 15 & 1.0 \\
\hline Non-axial & 20 & 19 & \\
\hline \multicolumn{4}{|l|}{ Depth of infiltrate } \\
\hline$<1 / 3$ of corneal thickness & 32 & 25 & 0.28 \\
\hline 1/3-2/3 of corneal thickness & 5 & 9 & \\
\hline Patients with hypopyon & 6 & 5 & \\
\hline Mean of hypopyon level (mm) & 0.57 & 0.54 & 1.0 \\
\hline
\end{tabular}

Table 3. Clinical signs and symptom scores at baseline.

\begin{tabular}{lccc}
\hline & $\begin{array}{c}\text { Fortified } \\
\text { Cefazolin/Amikacin }\end{array}$ & Levofloxacin & P-value \\
\hline $\begin{array}{l}\text { Scores for clinical signs } \\
\quad \text { Mean sign scores at baseline } \pm \text { SD }\end{array}$ & $3.72 \pm 2.09$ & $5.60 \pm 12.03$ & 0.99 \\
$\quad$ Mean of time to no signs \pm SD (days) & $12 \pm 1$ & $11 \pm 1$ & 0.37 \\
$\begin{array}{l}\text { Scores for clinical symptoms } \\
\quad \text { Mean symptom scores at baseline } \pm \text { SD }\end{array}$ & $9.54+4.29$ & $9.47+3.78$ & 0.85 \\
$\quad$ Mean of time to no symptoms \pm SD (days) & $13 \pm 1$ & $10 \pm 1$ & 0.38 \\
\hline
\end{tabular}


In the bacterial cultures, growth was detected in nine cases. Seven were Pseudomonas aeruginosa, one was stenotrophomonas, and one was co-infected with Pseudomonas aeruginosa and Enterobactor cloacae. Three specimens were positive for fungus and these patients were excluded from the study.

Table 4 shows results after treatment. After treatment, 61 out of 63 eyes (96.8\%) showed complete healing. There were no statistical differences for the resolution of keratitis $(\mathrm{p}=0.81)$ and mean time to heal between the two groups $(p=0.92)$. Further analysis of the mean time-duration to heal by the severity of the ulcer also showed no statistically significant differences between groups (Mild ulcers had a $\mathrm{p}$-value of 0.50. Severe ulcers had a p-value of 0.75 ). Concerning the time-duration for the clinical signs $(p=0.37)$ and symptoms $(p=0.38)$ to disappear, we did not observe any statistical differences between groups.

Interestingly, there were two patients from the levofloxacin group whose ulcers did not heal. At baseline, both of them had moderate corneal ulcers. The results from bacterial cultures showed that both of them had Pseudomonas aeruginosa. Since there was no clinical improvement after 72 hours of treatment, one of the patients decided to withdraw from the study. The other continued but eventually developed progressive corneal infiltration on day 7 . Treatment was immediately modified requiring the patient to frequently apply fortified Cefazolin and Amikacin. The ulcer eventually healed without yielding any serious complications.

During the study, there were no serious events caused by the disease or from the treatment regimens. Therefore, surgical interventions were not utilized, even in the two cases whose treatment regimen failed.

\section{Discussion}

Bacterial keratitis is one of the major devastating ocular conditions that quickly deteriorate the patient's vision [1]. Since the 1970s, there have been several RTCs looking at the efficacy of various secondgeneration fluoroquinolones (ofloxacin and ciprofloxacin), and fortified antibiotics for the treatment of bacterial keratitis [2-4, 9, 10]. Most studies showed no differences between the medications. Nevertheless, there have been reports of resistant bacterial strains to ofloxacin and ciprofloxacin that has led to the use of third- and fourth-generation fluoroquinolones eye drops [11-14].

It has been shown that fourth-generation fluoroquinolones is incapable of penetrating the corneal tissue and aqueous humor, resulting in significantly lower concentrations of gatifloxacin (0.3\%) compared to levofloxacin (1.5\%) in the targeted tissues [15]. This may be due to levofloxacin's solubility in water, which gives it the ability to easily penetrate the cornea, stroma tissue, and aqueous humor [16-20].

Table 4. Results after treatment.

\begin{tabular}{|c|c|c|c|}
\hline & $\begin{array}{c}\text { Fortified } \\
\text { Cefazolin/Amikacin } \\
(\mathbf{n}=37)\end{array}$ & $\begin{array}{l}\text { Levofloxacin } \\
\qquad(n=31)\end{array}$ & P-value \\
\hline Lost to follow-up & 3 & 2 & \\
\hline Complete healing of the ulcer & 34 & 27 & 0.81 \\
\hline Mild ulcer & 22 & 19 & \\
\hline Moderate ulcer & 12 & 8 & \\
\hline Unhealed ulcer & 0 & 2 & \\
\hline Mild ulcer & - & - & \\
\hline Moderate ulcer & - & 2 & \\
\hline \multicolumn{4}{|c|}{ Number of days it took the ulcer to completely heal } \\
\hline Mean \pm SD & $7 \pm 1$ & $7 \pm 1$ & 0.92 \\
\hline Mild ulcer & $6 \pm 1$ & $7 \pm 1$ & 0.50 \\
\hline Moderate ulcer & $10 \pm 1$ & $9 \pm 2$ & 0.75 \\
\hline \multicolumn{4}{|c|}{ Number of days it took the epithelial layer to heal } \\
\hline Mean \pm SD & $7 \pm 1$ & $8 \pm 1$ & 0.17 \\
\hline
\end{tabular}


In our study, we evaluated and compared the efficacy and safety of $0.5 \%$ topical levofloxacin to fortified board spectrum antibiotic, cefazolin, and amikacin, for the treatment of bacterial keratitis. The outcomes measured were resolution of keratitis, time it took for the ulcer to heal completely and adverse events. In patients with mild bacterial keratitis, both medications tested were equally effective. The ulcers in both treatment groups healed completely. The healing time between both groups was not statistically different. We did not detect any serious complications. Hence, we highly recommend the use of levofloxacin monotherapy for the treatment of mild bacterial keratitis.

As for patients with moderate corneal ulcers, we could demonstrate that Levofloxacin efficacy was comparable to the standard treatment. It should also be noted that two of our patients did not respond to Levofloxacin. However, in the moderate corneal ulcers, the healing-times between both groups were not significantly different. This further confirms that levofloxacin monotherapy is comparable to the fortified Cefazolin and Amikacin ophthalmic solution for the treatment of mild-to-moderate infectious bacterial keratitis.

We did not evaluate the efficacy of levofloxacin monotherapy in severe bacterial corneal ulcers. However, we must be careful for its use in these patients unless they are closely monitored. This note is based on other studies using second- and fourthgeneration monotherapy for the treatment of moderate-to-severe infectious keratitis that showed serious complications of visual loss and corneal perforation (4-18\%) [13, 14, 21].These studies suggest that the pathogens from severe bacterial corneal ulcers are more virulent and can easily become resistant to therapy if administered alone.Additional work is required to determine the efficacy and safety of levofloxacin monotherapy in patients with severe corneal ulcers.

We did not detect any serious adverse events in either treatment groups. Both drugs used in the study are considered safe even though other study reported corneal perforation [21]. In another study, corneal precipitations were observed to interfere with the healing process of the epithelial layer due to ciprofloxacin usage or other newer generations of fluoroquinolones such as gatifloxacin [22, 23].

In our study, Levofloxacin monotherapy should significantly reduce cost, as it is cheaper than the fortified Cefazolin and Amikacin ophthalmic solution. In addition, Levofloxacin seems to be more practical and easier to use since it does not require preparation before use. Also, it has been shown that Levofloxacin has fewer side effects compared to fortified Cefazolin and Amikacin ophthalmic solution. Therefore, this ease of use should increase the patient compliance to the treatment regimen. Hence, in resource-limited settings, Levofloxacin monotherapy would seem to be a better alternative compared to fortified Cefazolin and Amikacin ophthalmic solution in the treatment of mildto-moderate infectious bacterial keratitis.

Another significant limitation of the study was the number of positive cultures obtained from the corneal scrapings. Since two-thirds of our patients were diagnosed with mild bacterial keratitis, we could detect $17 \%$ cultural growth (nine bacterial isolates were detected). Therefore, we could perform the antibacterial susceptibility testing for all patients, which made it difficult to interpret the results. The antibacterial susceptibility testing would have helped us to predict the therapeutic response rate for fluoroquinolones for the treatment of bacterial keratitis. We attributed the low rate of culture due to the small amounts of tissue obtained from the corneal scrapings.

We noticed that out of the nine bacterial isolates, the bacterial spectrum was similar to the results from two other studies conducted in Thailand $[1,24]$. The most common pathogens found were Pseudomonas aeruginosa (78\%), Enterobactor cloacae, and Stenotrophomonas. In addition, we did not detect any gram-positive pathogens.

In conclusion, topical Levofloxacin monotherapy can be used for the treatment of mild to moderate bacterial corneal ulcers as an alternative treatment without developing any serious complications.

\section{Acknowledgement}

This article was supported in part by Daiichi, Thailand. There is no conflict of interest to report.

\section{References}

1. Boonpasart S, Kasetsuwan N, Puangsricharern V, Pariyakanok L, Jittpoonkusol T. Infectious keratitis at King Chulalongkorn Memorial Hospital: a 12-year retrospective study of 391 cases. J Med Assoc Thai. 2002; 85:217S-30S.

2. American Academy of Ophthalmology Vision Rehabilitation Panel. Preferred Practice Pattern ${ }^{\circledR}$ 
Guidelines. Bacterial Keratitis. San Francisco: American Academy of Ophthalmology; 2008. Available at: http://www.aao.org/ppp.

3. Leibowitz HM. Clinical evaluation of ciprofloxacin 0.3\% ophthalmic solution for treatment of bacterial keratitis. Am J Ophthalmol. 1991; 112:34S-47S.

4. O’Brien TP, Maguire MG, Fink NE, Alfonso E, McDonnell P. Efficacy of ofloxacin vs cefazolin and tobramycin in the therapy for bacterial keratitis: report from the Bacterial Keratitis Study Research Group. Arch Ophthalmol. 1995; 113:1257-65.

5. Ofloxacin Study Group. Ofloxacin monotherapy for the primary treatment of microbial keratitis: a doublemasked, randomized, controlled trial with conventional dual therapy. Ophthalmology. 1997; 104:1902-9.

6. Blondeau JM. Fluoroquinolones: mechanism of action, classification, and development of resistance. Surv Ophthalmol. 2004; 49:73S-8S.

7. Duggirala A, Joseph J, Sharma S, Nutheti R, Garg P, Das T. Activity of newer fluoroquinolones against gram-positive and gram-negative bacteria isolated from ocular infections: an in vitro comparison. Indian J Ophthalmol. 2007; 55:15-9.

8. Jones DB. Decision making in the management of microbial keratitis. Ophthalmology. 1981; 88:814-20.

9. Panda A, Ahuja R, Sastry SS. Comparison of topical $0.3 \%$ ofloxacin with fortified tobramycin plus cefazolin in the treatment of bacterial keratitis. Eye. 1999; 13:744-7.

10. Gangopadhyay N, Daniell M, Weih L, Taylor HR. Fluoroquinolone and fortified antibiotics for treating bacterial corneal ulcers. Br J Ophthalmol. 2000; 84: 378-84.

11. Goldstein MH, Kowalski RP, Gordon YJ. Emerging fluoroquinolones resistance in bacterial keratitis: a 5-year review. Ophthalmology. 1999; 106:1313-8.

12. Daniell M. Overview: initial antimicrobial therapy for microbial keratitis. Br J Ophthalmol. 2003; 87:1172-4.

13. Parmar P, Salman A, Kalavathy CM, Kaliamurthy J, Prasanth DA, Thomas PA, et al. Comparison of topical gatifloxacin $0.3 \%$ and ciprofloxacin $0.3 \%$ for the treatment of bacterial keratitis. Am J Ophthalmol. 2006; 141:282-6.

14. Constantinou M, Daniell M, Snibson GR, Vu HT, Taylor HR. Clinical efficacy of moxifloxacin in the treatment of bacterial keratitis: a randomized clinical trial. Ophthalmology. 2007; 114:1622-9.
15. Holland EJ, McCarthy M, Holland S. The ocular penetration of levofloxacin $1.5 \%$ and gatifloxacin $0.3 \%$ ophthalmic solutions in subjects undergoing corneal transplant surgery. Curr Med Res Opin. 2007; 23: 2955-60.

16. Kowalski RP, Dhaliwal DK, Karenchak LM, Romanowski EG, Mah FS, Ritterband DC, et al. Gatifloxacin and moxifloxacin: an in vitro susceptibility comparison to levofloxacin, ciprofloxacin, and ofloxacin using bacterial keratitis isolates. Am J Ophthalmol. 2003; 136:500-5.

17. Yamada M, Mochizuki H, Yamada K, Kawai M, Mashima Y. Aqueous humor levels of topically applied levofloxacin in human eyes. Curr Eye Res. 2002; 24: 403-6.

18. Colin J, Simonpoli S, Geldsetzer K, Ropo A. Corneal penetration of levofloxacin into the human aqueous humour: a comparison with ciprofloxacin. Acta Ophthalmol Scand. 2003; 81:611-3.

19. Bucci FA Jr. An in vivo study comparing the ocular absorption of levofloxacin and ciprofloxacin prior to phacoemulsification. Am J Ophthalmol. 2004; 137: 308-12.

20. Healy DP, Holland EJ, Nordlund ML, Dunn S, Chow C, Lindstrom RL, et al. Concentrations of levofloxacin, ofloxacin, and ciprofloxacin in human corneal stromal tissue and aqueous humor after topical administration. Cornea. 2004; 23:255-63.

21. Mallari PL, McCarty DJ, Daniell M, Taylor H. Increased incidence of corneal perforation after topical fluoroquinolone treatment for microbial keratitis. Am J Ophthalmol. 2001; 131:131-3.

22. Tanhehco TY, Chiavetta SV 3rd, Lee PP, Fowler AM, Afshari NA. “Cracked-mud” ciprofloxacin precipitates on a corneal graft. Ophthalmic Surg Lasers Imaging. 2005; 36:252-3.

23. Awwad ST, Haddad W, Wang MX, Parmar D, Conger $\mathrm{D}$, Cavanagh HD. Corneal intrastromal gatifloxacin crystal deposits after penetrating keratoplasty. Eye Contact Lens. 2004; 30:169-72.

24. Erjongmanee S, Kasetsuwan N, Phusitphoykai N, Puangsricharern V, Pariyakanok L. Clinical evaluation of ophthalmic lomefloxacin $0.3 \%$ in comparison with fortified cefazolin and gentamicin ophthalmic solutions in the treatment of presumed bacterial keratitis. J Med Assoc Thai. 2004; 87:83S-90S. 